\title{
Health benefits of fermented milk containing Bifidobacterium bifidum YIT 10347 on gastric symptoms in adults
}

\author{
A. Gomi, ${ }^{* 1}$ T. lino, ${ }^{*}$ C. Nonaka, $†$ K. Miyazaki, ${ }^{*}$ and F. Ishikawa* \\ *Yakult Central Institute, Kunitachi, Tokyo 186-8650, Japan \\ †Faculty of Research and Development, Yakult Honsha, Shimbashi, Tokyo 105-8660, Japan
}

\begin{abstract}
We conducted a preliminary open trial (trial 1) and a double-blind, placebo-controlled, crossover trial (trial 2) to examine how fermented milk containing the probiotic Bifidobacterium bifidum YIT 10347 affects gastric and lower abdominal symptoms in adults taking no medication. In trial 1, subjects with or without gastric and lower abdominal symptoms ingested fermented milk containing B. bifidum YIT 10347 daily for 2 wk. In trial 2, subjects with gastric symptoms ingested fermented milk containing B. bifidum YIT 10347 (active preparation) or placebo daily for $2 \mathrm{wk}$, followed by crossover for 3 wk after a washout period. Before (baseline) and 1 and 2 wk after ingestion, subjects completed a questionnaire. In trial 1 (305 subjects), the prevalence of gastric and lower abdominal symptoms was 46 and $58 \%$, respectively, at baseline. Ingestion of B. bifidum YIT 10347 significantly decreased the prevalence of gastric and lower abdominal symptoms from 45 to $33 \%$ at $1 \mathrm{wk}$ and to $28 \%$ at $2 \mathrm{wk}$, and from 57 to $40 \%$ at 2 wk, respectively. In subjects with gastric symptoms at baseline, the average gastric symptom score per subject significantly decreased by 0.9 at 1 wk and 1.2 at 2 wk. In trial 2 (27 subjects), ingestion of the active preparation significantly decreased the average gastric symptoms score per subject by 1.0 at 1 wk and 1.1 at 2 wk, but ingestion of placebo milk had no effect. No side effects were reported by any subjects in either trial. We conclude that fermented milk containing B. bifidum YIT 10347 has the potential to provide health benefits by alleviating gastric symptoms in subjects taking no medication.
\end{abstract}

Key words: Bifidobacterium bifidum, fermented milk, gastric symptoms, questionnaire

\footnotetext{
Received November 25, 2014.

Accepted December 12, 2014

${ }^{1}$ Corresponding author: atsushi-gomi@yakult.co.jp
}

\section{INTRODUCTION}

Functional gastrointestinal (GI) disorders, such as functional dyspepsia (FD) and irritable bowel syndrome (IBS), are characterized by chronic or recurrent pain and stomach and lower abdominal symptoms. Despite the lack of structural anomalies, patients with FD may display upper abdominal pain, bloating, fullness, and tenderness on palpation (Talley et al., 2005; Saad and Chey, 2006; Tack et al., 2006), and patients with IBS may display lower abdominal pain, diarrhea, constipation, and bloating (Longstreth et al., 2006; Mayer, 2008). Functional dyspepsia and IBS are estimated to occur in about 15\% (Saad and Chey, 2006) and 10 to 15\% (Boivin, 2001; Quigley et al., 2006), respectively, of the general population in Western countries, and the underlying mechanisms remain to be fully clarified. Some associations between FD and Helicobacter pylori infection have been considered in the elderly in Western countries (Talley et al., 2005; Saad and Chey, 2006). However, the incidence of gastro-esophageal reflux diseases and FD is increasing considerably in younger Japanese adults (Watanabe et al., 2007), who have a low infection rate of $H$. pylori. Because FD and IBS are frequently accompanied by mental disorders, such as depression, the prevention and improvement of FD and IBS symptoms play a pivotal role in the maintenance of health-related quality of life. Additionally, the number of healthy subjects who occasionally feel gastric and lower abdominal symptoms is increasing. Typical symptoms include abdominal pain, stomach heaviness, and acidic regurgitation (as in FD), and difficult defecation, occasional diarrhea, and lower abdominal distension (as in IBS). Such individuals expect to receive food supplements or functional foods with or without probiotics rather than medical treatment, because their symptoms are mild and infrequent.

Probiotics are living microorganisms that, when consumed in adequate amounts, confer a health benefit on the host (Fuller, 1991). Several probiotic strains of lactobacilli and bifidobacteria have been used in dairy fermented foods or as food supplements, and are habitu- 
ally ingested around the world. There is much evidence that some probiotics have many beneficial health effects, including the suppression of harmful bacteria and metabolites in the gut, inhibition of bowel disorders (Cain and Karpa, 2011), immune modulation (Isolauri et al., 2001), and antiallergy, antiinflammation, and anticancer effects (Reid and Hammond, 2005; Miyazaki and Matsuzaki, 2008; Umesaki et al., 2012).

Studies have shown that selected probiotics provide benefits not only in suppressing the growth and activity of H. pylori in vitro and in vivo (Sakamoto et al., 2001; Sgouras et al., 2004; Sykora et al., 2005) but also in alleviating symptoms of IBS (Agrawal et al., 2009; Moayyedi et al., 2010; Horvath et al., 2011) and upper and lower GI symptoms (Waller et al., 2011) in clinical trials. Oral administration of $B$. bifidum (strain YIT 4007) promotes repair and protective activities in the gastric ulcer rat model (Nagaoka et al., 1994), reduces the severity of peptic ulcer to a mild state, and inhibits $H$. pylori infection in the gastric mucosa in patients (Yamamoto et al., 1994). However, few studies have examined the effects of fermented milk containing probiotics on FD and gastric symptoms in healthy adults.

Therefore, to establish a new strategy using probiotics for the prevention and improvement of FD, we conducted 2 trials - a preliminary open trial (trial 1) and a double-blind, placebo-controlled, crossover trial (trial 2) - to examine the effects of fermented milk containing B. bifidum YIT 10347 on gastric symptoms in healthy adults taking no medication.

\section{MATERIALS AND METHODS}

\section{Subjects}

In trial 1, 323 volunteers (167 men and 156 women) were enrolled, with a mean ( \pm SD) age of $50.6 \pm 7.4 \mathrm{yr}$ (range, 33-84 yr) who either did or did not experience gastric or lower abdominal symptoms. In trial 2, 30 volunteers (26 men and 4 women) were enrolled, with a mean $( \pm \mathrm{SD})$ age of $35.3 \pm 11.3 \mathrm{yr}$ (range, $21-58 \mathrm{yr}$ ) who sometimes experienced gastric symptoms.

During both trials, ingestion compliance and changes in health condition were self-recorded in a diary. All subjects were free from medication known to affect GI symptoms for 1 mo before and during the trial; avoided the intake of other fermented milk, probiotic, and prebiotic products; and did not change their lifestyle routine. Subjects who did not meet these criteria were excluded from the analysis. Trials 1 and 2 were conducted in accordance with all appropriate laws, regulations, and policies regarding the use of human subjects in research, and were approved by the Ethics Committees of Yakult Central Institute for Microbiological Research. Signed informed consent was obtained from all subjects before enrollment.

\section{Test Beverage}

Fermented milk containing B. bifidum YIT 10347 (the active preparation) was prepared by anaerobic culture of B. bifidum YIT 10347 and Streptococcus thermophilus YIT 2021 in sterilized milk at $37^{\circ} \mathrm{C}$ for $24 \mathrm{~h}$ (Miki et al., 2007). Both strains were obtained from the Culture Collection Laboratory at Yakult Central Institute (Tokyo, Japan). The placebo was prepared from an anaerobic culture of Strep. thermophilus YIT 2021 in sterilized milk at $37^{\circ} \mathrm{C}$ for $24 \mathrm{~h}$; the flavor was as similar as possible to the active preparation. These samples were supplied as identically packaged beverages (100 $\mathrm{mL} /$ bottle) to subjects at intervals of $1 \mathrm{wk}$. The active and placebo beverages were coded such that researchers did not know the order in which subjects received active and placebo beverages. The active preparation contained more than $1 \times 10^{7} \mathrm{cfu} / \mathrm{mL}$ of B. bifidum YIT 10347 and approximately $1 \times 10^{7} \mathrm{cfu} / \mathrm{mL}$ of Strep. thermophilus YIT 2021. The placebo contained more than $1 \times 10^{7} \mathrm{cfu} / \mathrm{mL}$ of Strep. thermophilus YIT 2021 . The counts (colony-forming units) of B. bifidum YIT 10347 or Strep. thermophilus YIT 2021 in samples was determined by culturing samples on plates containing TOS (transgalactosylated oligosaccharides) propionate agar medium (Yakult Pharmaceutical Industry Co. Ltd., Tokyo, Japan) or Difco Lactobacilli MRS (de Man, Rogosa, and Sharpe) agar (Becton Dickinson Co., Sparks, MD).

\section{Trial Design}

In trial 1 , the preliminary open trial, 323 adult subjects drank $100 \mathrm{~mL} / \mathrm{d}$ of fermented milk containing B. bifidum YIT 10347 for 2 wk. Trial 2, the doubleblind, placebo-controlled, crossover trial, comprised a preingestion period of $1 \mathrm{wk}$, an ingestion period of 2 wk, a washout period of $3 \mathrm{wk}$, and a crossover ingestion period of 2 wk. Before the preingestion period, 30 subjects were divided into 2 groups matched for age, weight, height, and body mass index. During the ingestion period, one group ingested $100 \mathrm{~mL} / \mathrm{d}$ of fermented milk containing B. bifidum YIT 10347 (active preparation) and the other group ingested $100 \mathrm{~mL} / \mathrm{d}$ of placebo.

In both trials, a questionnaire was completed before (baseline) and 1 and 2 wk after ingestion of the beverage. 


\section{Questionnaire}

In trial 1, the modified gastrointestinal symptom rating scale (Svedlund et al., 1988) was used. The scale consisted of 14 questions with regard to the following 8 gastric and 6 lower abdominal symptoms and 4 frequencies: A1, acid regurgitation; A2, heartburn; A3, abdominal pains; A4, stomach heaviness; A5, no appetite; A6, nausea or vomiting; A7, burp; A8, repeated abdominal pain or discomfort; A9, difficult defecation; A10, feeling of incomplete defecation; A11, occasional diarrhea; A12, lower abdominal distension; A13, borborygmus; and A14 lower abdominal pain. Frequency was determined as follows: none $=$ score 0 ; occasionally $(1$ or $2 \mathrm{~d} / \mathrm{wk})=$ score 1 ; sometimes $(3$ to $5 \mathrm{~d} / \mathrm{wk})=$ score 2 ; and frequently $(6$ or $7 \mathrm{~d} / \mathrm{wk})=$ score 3 . Questions A1 to A8 and A9 to A14 were used to analyze symptoms of the stomach and intestine, respectively.

In trial 2, a modified F-scale (Watanabe et al., 2007) was used to analyze gastric symptoms. The modified F-scale consisted of questions regarding the following 10 gastric symptoms, 3 levels of severity, and 3 frequencies: B1, acidic regurgitation; B2, heartburn; B3, midnight abdominal pain; B4, fasting abdominal pain; B5, abdominal distension; B6, stomach heaviness; B7, no appetite; B8, nausea or vomiting; B9, burp; and B10, repeat of abdominal pain or discomfort. Severity was measured as follows: none $=$ score 0 ; mild $=$ score 1 ; and severe $=$ score 2 . Frequency was determined as follows: none $=$ score 0 ; sometimes $(1$ to $4 \mathrm{~d} / \mathrm{wk})=$ score 1 ; and frequently $(5$ to $7 \mathrm{~d} / \mathrm{wk})=$ score 2 . The overall score for each symptom was calculated by multiplying severity by frequency, and the total scores of all symptoms were summed.

For gastric and lower abdominal symptoms, prevalence was calculated by dividing the number of subjects with one or more symptoms by the total number of subjects analyzed. The average number of symptoms was calculated by dividing the number of symptoms by the number of subjects with one or more symptoms. The average scores of gastric and lower abdominal symptoms were calculated by dividing the respective summed scores by the total number of subjects analyzed.

\section{Statistical Analysis}

Subjects who did not answer one or more items on the questionnaires were excluded from the analysis. The score was expressed as mean \pm standard error. The chisquared test and Wilcoxon signed-rank test were used to analyze changes in the prevalence and differences of the score, respectively; $P<0.05$ was considered statistically significant.

\section{RESULTS}

\section{Trial 1}

First, a preliminary open trial was conducted, in which 323 subjects ingested $100 \mathrm{~mL} / \mathrm{d}$ of fermented milk containing B. bifidum YIT 10347 for 2 wk and completed a questionnaire regarding 14 symptoms associated with gastric and lower abdominal symptoms, before (baseline) and 1 and 2 wk after ingestion. Ingestion compliance was more than 95\%. However, 18 and 12 subjects who did not answer one or more items on the gastric or intestinal questionnaire, respectively, were excluded. Therefore, 305 and 311 subjects were included in the following analysis.

Figure 1 shows the prevalence of and change in gastric symptoms. At baseline, 139 subjects reported one or more gastric symptoms, demonstrating that the prevalence of gastric symptoms was $45 \%$. The ingestion of fermented milk containing B. bifidum YIT 10347 decreased the prevalence from 45 to $33 \%(P<0.05)$ after $1 \mathrm{wk}$ and to $28 \%(P<0.01)$ after 2 wk. Second, subgroup analysis was performed for the 149 subjects with gastric symptoms at baseline. Figures $2 \mathrm{~A}$ and $2 \mathrm{~B}$ show the changes in the average number and average score, respectively, for gastric symptoms per subject. The ingestion of B. bifidum YIT 10347 decreased the average number of gastric symptoms per subject by $0.8(P<0.001)$ and $1.1(P<0.001)$ at 1 and $2 \mathrm{wk}$, respectively, and decreased the average gastric score per subject by $0.9(P<0.001)$ and $1.2(P<0.01)$ at 1 and $2 \mathrm{wk}$, respectively. In addition, ingestion for $2 \mathrm{wk}$ reduced the prevalence of 5 gastric symptoms: stomach heaviness $(P<0.0001)$, burp $(P<0.001)$, no appetite $(P<0.01)$, repeat of abdominal pain or discomfort $(P$ $<0.05)$, and acid regurgitation $(P<0.05)$.

Figure 3 shows the prevalence of and change in lower abdominal symptoms. At baseline, 177 subjects reported one or more lower abdominal symptoms, showing that the prevalence of lower abdominal symptoms was $57 \%$. The ingestion of fermented milk containing $B$. bifidum YIT 10347 decreased the prevalence from 57 to $47 \%$ at $1 \mathrm{wk}(P<0.05)$ and to $40 \%(P<0.01)$ at 2 wk. A subgroup analysis was performed in the 177 subjects with lower abdominal symptoms at baseline. Figures $4 \mathrm{~A}$ and $4 \mathrm{~B}$ show changes in the average number and the average score, respectively, for lower abdominal symptoms per subject. The ingestion of B. bifidum YIT 10347 decreased the average number of lower abdominal symptoms per subject by $0.6(P<0.001)$ and 0.9 $(P<0.001)$ at 1 and $2 \mathrm{wk}$, respectively, and decreased the average lower abdominal symptom score per subject by $0.8(P<0.001)$ and $1.1(P<0.01)$ at 1 and 2 wk, respectively. Furthermore, ingestion of B. bifidum 


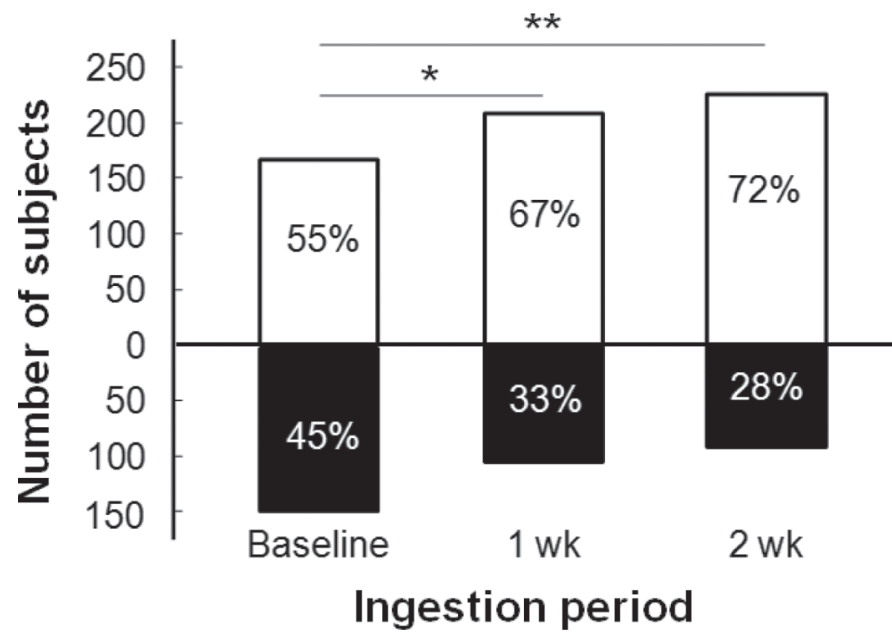

Figure 1. The ingestion of fermented milk containing Bifidobacterium bifidum YIT 10347 reduced the prevalence of gastric symptoms in adults. White bars $=$ no gastric symptoms; black bars $=$ with gastric symptoms (one or more symptoms). Percentage shows the prevalence, which was calculated by dividing the number of subjects with gastric symptoms by the total number of subjects ( 305 subjects) enrolled in the analysis with chi-squared test $\left({ }^{*} P<0.05\right.$, $\left.{ }^{* *} P<0.01\right)$.

YIT 10347 for 2 wk reduced the prevalence of 4 lower abdominal symptoms: occasional diarrhea $(P<0.01)$, difficult defecation $(P<0.01)$, lower abdominal distension $(P<0.01)$, and feeling of incomplete defecation $(P<0.05)$.

\section{Trial 2}

To clarify the effect of ingestion of fermented milk containing B. bifidum YIT 10347 on gastric symptoms, we conducted a double-blind, placebo-controlled, cross- over trial (trial 2). Thirty subjects with gastric symptoms ingested fermented milk containing B. bifidum YIT 10347 (active preparation) or placebo milk daily for $2 \mathrm{wk}$, followed by crossover for 3 wk after a washout period, and answered a questionnaire before (baseline) and 1 and 2 wk after ingestion. Two subjects were excluded because they used a gastrointestinal drug, and 1 subject dropped out for personal reasons. The ingestion compliance was $>95 \%$ among the remaining 27 subjects who were included in the analysis.

Figure 5 shows changes in the average gastric symptom score in the active and placebo groups. Ingestion of the active preparation decreased the average gastric score per subject by $1.0(P<0.001)$ and $1.1(P<0.001)$ at 1 and 2 wk [symptom score was 1.74 (baseline), 0.78 (1 wk), and 0.67 (2 wk)], respectively, but the ingestion of placebo had no effect [symptom score was 1.59 (baseline), 1.11 (1 wk), and 1.11 (2 wk)]. Meanwhile, the effects of ingestion of B. bifidum YIT 10347 on the prevalence of symptoms, the number of subjects with gastric symptoms [active: 18 (baseline), 11 (1 wk), 11 (2 wk); placebo: 16 (baseline), 10 (1 wk), 12 (2 wk)], and the number of symptoms per subject [active: 2.6 (baseline), 1.9 (1 wk), 1.6 (2 wk); placebo: 2.7 (baseline), 2.3 (1 wk), 1.9 (2 wk)] were not significant.

In both trials, no subjects experienced abnormal changes or adverse effects, such as diarrhea, constipation, or bloating.

\section{DISCUSSION}

To establish a new strategy using probiotics for the prevention and improvement of FD, we conducted 2 tri-
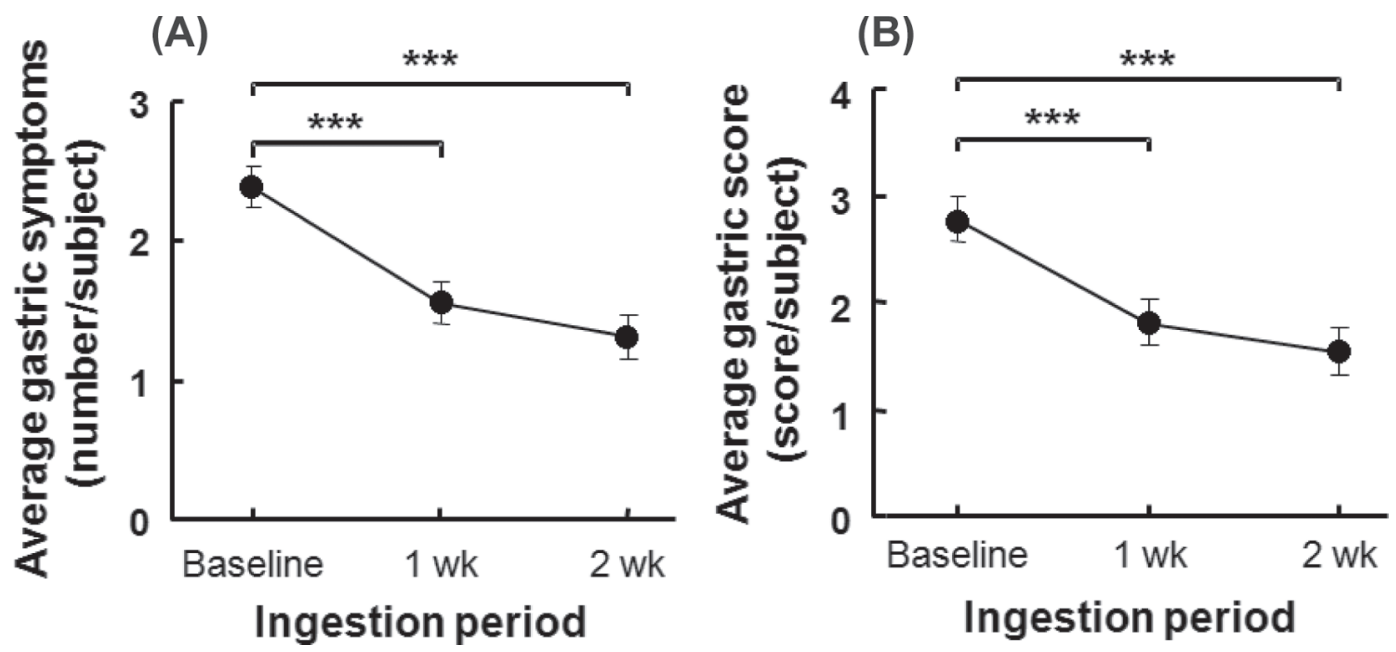

Figure 2. The ingestion of fermented milk containing Bifidobacterium bifidum YIT 10347 reduced both average number of intestinal symptoms (A) and average gastric score (B) in subjects with gastric symptoms (one or more symptoms) at baseline. Error bars represent SE. Data from 149 subjects were analyzed by Wilcoxon signed-rank test $(* * * P<0.001)$. 


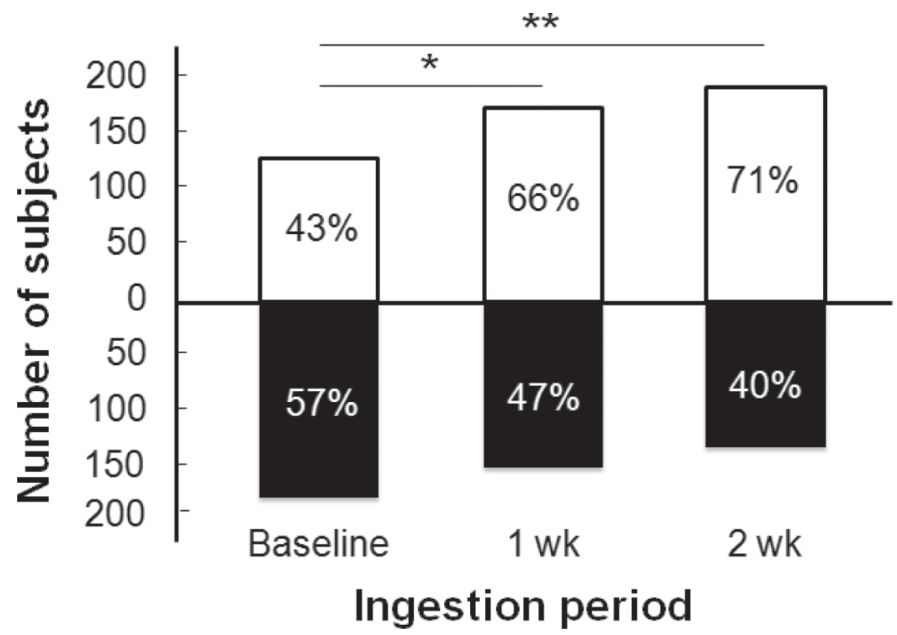

Figure 3. The ingestion of fermented milk containing Bifidobacterium bifidum YIT 10347 reduced the prevalence of lower abdominal symptoms in adults. White bars $=$ no lower abdominal symptoms; black bars $=$ with lower abdominal symptoms (one or more symptoms). Percentage shows the prevalence, which was calculated by dividing the number of subjects with lower abdominal symptoms by the total number of subjects (311 subjects) enrolled in the analysis with chi-squared test $\left({ }^{*} P<0.05,{ }^{* *} P<0.01\right)$.

als: a preliminary open trial including subjects with or without gastric and lower abdominal symptoms and a double-blind, placebo-controlled, crossover trial including subjects with gastric symptoms but no medication. The effects of fermented milk containing B. bifidum YIT 10347 on GI symptoms were examined using questionnaires.

The results of the preliminary open trial demonstrated that the daily ingestion of fermented milk containing B. bifidum YIT 10347 decreased the prevalence, aver- age score, and average numbers of symptoms of gastric and lower abdominal symptoms. The findings of the open trial indicate that the ingestion of fermented milk containing B. bifidum YIT 10347 may provide health benefits to subjects with gastric or lower abdominal symptoms. The results indicate that the potential for fermented milk containing B. bifidum YIT 10347 to improve gastric symptoms is higher than that for intestinal symptoms, because the prevalence of gastric symptoms was reduced at $1 \mathrm{wk}$, more symptoms were improved in the stomach than the intestine, and the lowest $P$-value in the reduction of prevalence was found for "stomach heaviness" after ingestion of B. bifidum YIT 10347 for 2 wk. Moreover, the results of the double-blind, placebo-controlled, crossover trial demonstrated that the average score was lowered by the daily ingestion of fermented milk containing B. bifidum YIT 10347 but not placebo in subjects with gastric symptoms. Furthermore, daily ingestion of the fermented milk appears to have health benefits in regard to gastric symptoms.

Previous clinical trials have demonstrated that daily ingestion of fermented milk containing B. bifidum YIT 10347 improves H. pylori-associated gastritis, inhibits H. pylori activity (Miki et al., 2007), and stimulates gastric emptying (Urita et al., 2008). It has also been demonstrated that B. bifidum YIT 10347 has potential to inhibit $H$. pylori activity and the induction of IL-8 in H. pylori-infected gastric epithelial cells (Miki et al., $2007)$ via the regulation of nuclear factor $\kappa \mathrm{B}(\mathrm{NF}-\kappa \mathrm{B})$ signaling pathways (Shirasawa et al., 2010), to alleviate drug-induced acute injury due to the stimulated production of gastroprotective mucin in rat stomach (Gomi et al., 2013), and to adhere highly to both human gastric epithelial cells (GCIY cells) and mucin
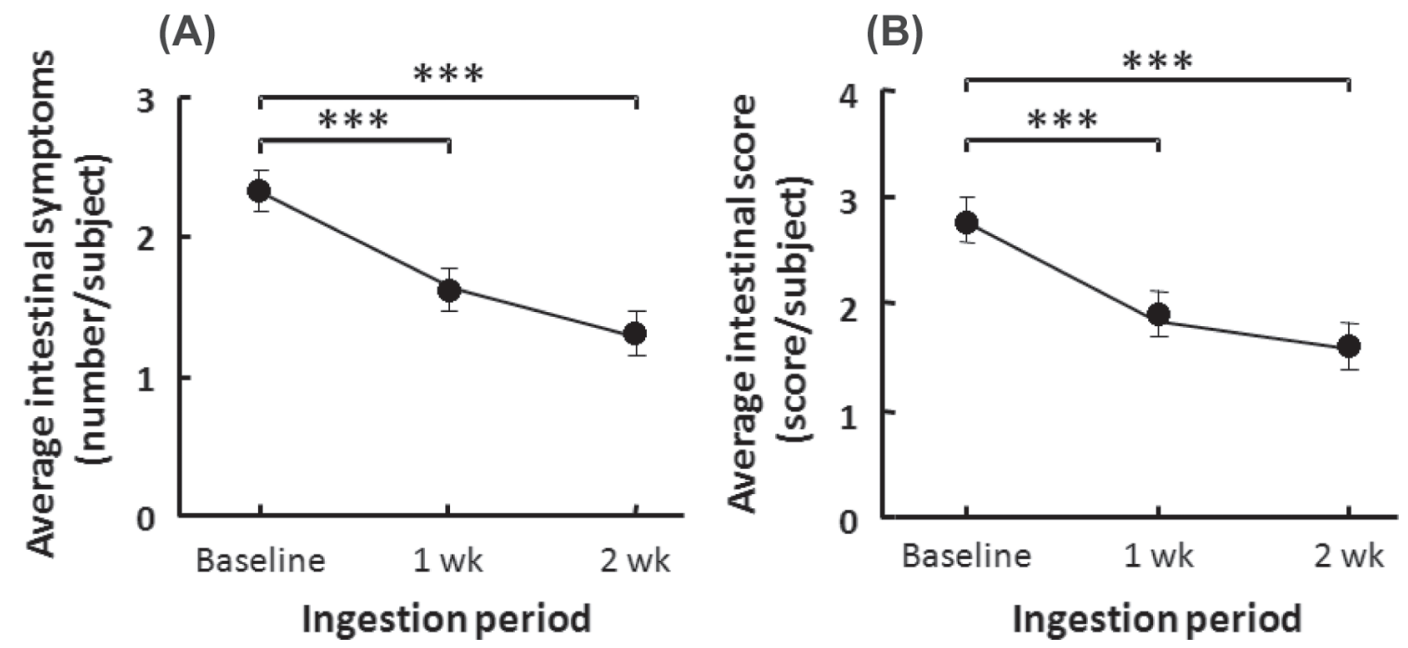

Figure 4. The ingestion of fermented milk containing Bifidobacterium bifidum YIT 10347 reduced both average number of intestinal symptoms (A) and average intestinal score (B) in subjects with lower abdominal symptoms (one or more symptoms) at baseline. Error bars represent SE. Data from 177 subjects were analyzed by Wilcoxon signed-rank test $(* * * P<0.001)$. 


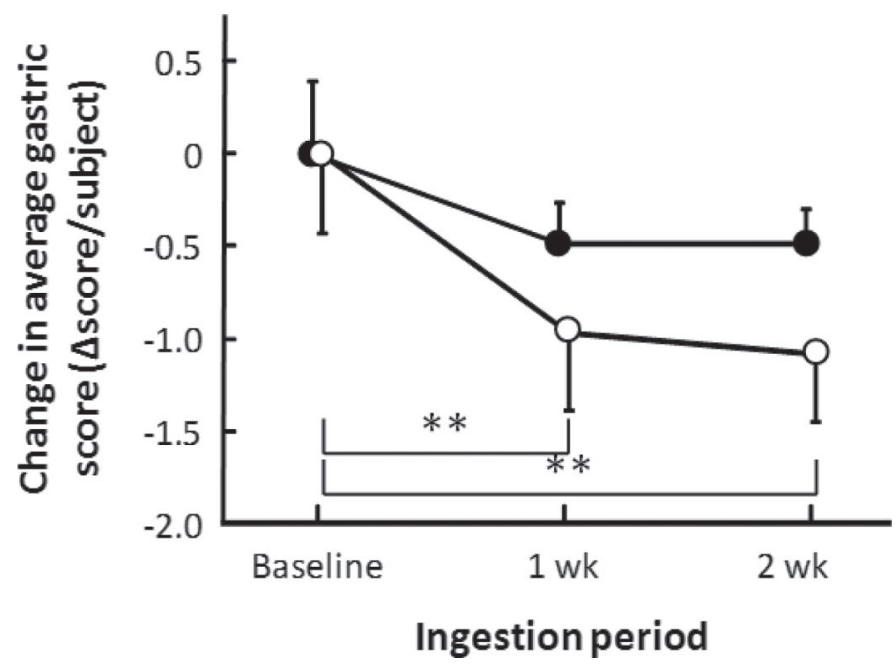

Figure 5. The average gastric score was reduced by the ingestion of fermented milk containing Bifidobacterium bifidum YIT 10347 (active preparation; O) but not placebo (-) in a double-blind, placebo-controlled, crossover trial. Error bars represent SE. Data from 27 subjects were analyzed by Wilcoxon signed-rank test $(* * P<0.01)$.

layer in vitro [A. Gomi, H. Shibahara-Sone, T. Iino, Y. Shimakawa, K. Miyazaki and F. Ishikawa (Yakult Central Institute), unpublished data]. We suggest that some health benefits of B. bifidum YIT 10347 on the stomach are based on its increased ability to adhere to epithelial cells, the stimulated production of gastroprotective mucin, and other possible factors such as GI or neuropeptide hormones that regulate gastric functions.

In trial 1 , lower abdominal symptoms were improved after ingestion of B. bifidum YIT 10347 fermented milk. It has been reported that B. bifidum YIT 10347 can reach the intestines alive (Fujimoto and Watanabe, 2013). Therefore, consumption of B. bifidum YIT 10347 fermented milk may ameliorate the intestinal environment, leading to improved lower abdominal symptoms. Measurement of changes in gut microbiota after consumption of B. bifidum YIT 10347 fermented milk in a future study would be helpful in further elucidating this connection.

There are 2 types of gastric symptoms in FD. First, abdominal pain is caused by over-production of gastric acid or under-protection of gastric acid; second, abdominal symptoms occur after eating due to the lowering of gastric emptying. It has been reported that gastric emptying is reduced in FD patients (Kamino et al., 2008; Matsumoto et al., 2008). Therefore, we speculate that the stimulated production of gastroprotective mucin by B. bifidum YIT 10347 is involved in the alleviation of gastric pain, and stimulation of gastric emptying by B. bifidum YIT 10347 is involved in the alleviation of uncomfortable stomach symptoms after eating.
In conclusion, the ingestion of fermented milk containing B. bifidum YIT 10347 has the potential to provide health benefits by alleviating gastric symptoms in subjects taking no medication. Further studies are necessary clarify the benefits of B. bifidum YIT 10347 in more subjects with FD and GI symptoms.

\section{ACKNOWLEDGMENTS}

We are grateful to Naomi Harima-Mizusawa, Harue Shibahara-Sone, Norie Onodera-Masuoka, and Mitsuyoshi Kano (Yakult Central Institute) for technical help and discussions.

\section{REFERENCES}

Agrawal, A., L. A. Houghton, J. Morris, B. Reilly, D. Guyonnet, N. Goupil Feuillerat, A. Schlumberger, S. Jakob, and P. J. Whorwell. 2009. Clinical trial: The effects of a fermented milk product containing Bifidobacterium lactis DN-173 010 on abdominal distension and gastrointestinal transit in irritable bowel syndrome with constipation. Aliment. Pharmacol. Ther. 29:104-114.

Boivin, M. 2001. Socioeconomic impact of irritable bowel syndrome in Canada. Can. J. Gastroenterol. 15(Suppl. B):8B-11B.

Cain, A. M., and K. D. Karpa. 2011. Clinical utility of probiotics in inflammatory bowel disease. Altern. Ther. Health Med. 17:72-79.

Fujimoto, J., and K. Watanabe. 2013. Quantitative detection of viable Bifidobacterium bifidum BF-1 cells in human feces by using propidium monoazide and strain-specific primers. Appl. Environ. Microbiol. 79:2182-2188.

Fuller, R. 1991. Probiotics in human medicine. Gut 32:439-442.

Gomi, A., N. Harima-Mizusawa, H. Shibahara-Sone, M. Kano, K. Miyazaki, and F. Ishikawa. 2013. Effect of Bifidobacterium bifidum BF-1 on gastric protection and mucin production in an acute gastric injury rat model. J. Dairy Sci. 96:832-837.

Horvath, A., P. Dziechciarz, and H. Szajewska. 2011. Meta-analysis: Lactobacillus rhamnosus GG for abdominal pain-related functional gastrointestinal disorders in childhood. Aliment. Pharmacol. Ther. 33:1302-1310.

Isolauri, E., Y. Sutas, P. Kankaanpaa, H. Arvilommi, and S. Salminen. 2001. Probiotics: Effects on immunity. Am. J. Clin. Nutr. 73(Suppl.):444S-450S.

Kamino, D., N. Manabe, J. Hata, K. Haruma, S. Tanaka, and K. Chayama. 2008. Long-term ultrasonographic follow-up study of gastric motility in patients with functional dyspepsia. J. Clin. Biochem. Nutr. 42:144-149.

Longstreth, G. F., W. G. Thompson, W. D. Chey, L. A. Houghton, F. Mearin, and R. C. Spiller. 2006. Functional bowel disorders. Gastroenterology 130:1480-1491.

Matsumoto, Y., M. Ito, D. Kamino, S. Tanaka, K. Haruma, and K. Chayama. 2008. Relation between histologic gastritis and gastric motility in Japanese patients with functional dyspepsia: Evaluation by transabdominal ultrasonography. J. Gastroenterol. 43:332-337.

Mayer, E. A. 2008. Clinical practice. Irritable bowel syndrome. N. Engl. J. Med. 358:1692-1699.

Miki, K., Y. Urita, F. Ishikawa, T. Iino, H. Shibahara-Sone, R. Akahoshi, S. Mizusawa, A. Nose, D. Nozaki, K. Hirano, C. Nonaka, and T. Yokokura. 2007. Effect of Bifidobacterium bifidum fermented milk on Helicobacter pylori and serum pepsinogen levels in humans. J. Dairy Sci. 90:2630-2640.

Miyazaki, K., and T. Matsuzaki. 2008. Health properties of milk fermented with Lactobacillus casei strain Shirota (LcS). Pages 165-208 in Handbook of Fermented Functional Foods, CRC Press, Boca Raton, FL. 
Moayyedi, P., A. C. Ford, N. J. Talley, F. Cremonini, A. E. FoxxOrenstein, L. J. Brandt, and E. M. Quigley. 2010. The efficacy of probiotics in the treatment of irritable bowel syndrome: A systematic review. Gut 59:325-332.

Nagaoka, M., S. Hashimoto, T. Watanabe, T. Yokokura, and Y. Mori. 1994. Anti-ulcer effects of lactic acid bacteria and their cell wall polysaccharides. Biol. Pharm. Bull. 17:1012-1017.

Quigley, E. M., G. R. Locke, S. Mueller-Lissner, L. G. Paulo, G. N. Tytgat, I. Helfrich, and E. Schaefer. 2006. Prevalence and management of abdominal cramping and pain: A multinational survey. Aliment. Pharmacol. Ther. 24:411-419.

Reid, G., and J. A. Hammond. 2005. Probiotics. Some evidence of their effectiveness. Can. Fam. Physician 51:1487-1493.

Saad, R. J., and W. D. Chey. 2006. Review article: Current and emerging therapies for functional dyspepsia. Aliment. Pharmacol. Ther. 24:475-492.

Sakamoto, I., M. Igarashi, K. Kimura, A. Takagi, T. Miwa, and Y. Koga. 2001. Suppressive effect of Lactobacillus gasseri OLL 2716 (LG21) on Helicobacter pylori infection in humans. J. Antimicrob. Chemother. 47:709-710.

Sgouras, D., P. Maragkoudakis, K. Petraki, B. Martinez-Gonzalez, E. Eriotou, S. Michopoulos, G. Kalantzopoulos, E. Tsakalidou, and A. Mentis. 2004. In vitro and in vivo inhibition of Helicobacter pylori by Lactobacillus casei strain Shirota. Appl. Environ. Microbiol. 70:518-526.

Shirasawa, Y., H. Shibahara-Sone, T. Iino, and F. Ishikawa. 2010. Bifidobacterium bifidum BF-1 suppresses Helicobacter pylori-induced genes in human epithelial cells. J. Dairy Sci. 93:4526-4534.

Svedlund, J., I. Sjodin, and G. Dotevall. 1988. GSRS-A clinical rating scale for gastrointestinal symptoms in patients with irritable bowel syndrome and peptic ulcer disease. Dig. Dis. Sci. 33:129-134.

Sykora, J., K. Valeckova, J. Amlerova, K. Siala, P. Dedek, S. Watkins, J. Varvarovska, F. Stozicky, P. Pazdiora, and J. Schwarz. 2005. Effects of a specially designed fermented milk product containing probiotic Lactobacillus casei DN-114 001 and the eradication of $H$. pylori in children: A prospective randomized double-blind study. J. Clin. Gastroenterol. 39:692-698.

Tack, J., N. J. Talley, M. Camilleri, G. Holtmann, P. Hu, J. R. Malagelada, and V. Stanghellini. 2006. Functional gastroduodenal disorders. Gastroenterology 130:1466-1479.

Talley, N. J., and N. Vakil., and Practice Parameters Committee of the American College of Gastorenterology. 2005. Guidelines for the management of dyspepsia. Am. J. Gastroenterol. 100:2324-2337.

Umesaki, Y., K. Matsumoto, T. Asahara, M. Onoue, K. Kawakami, M. Nanno, K. Shida, S. Matsumoto, F. Ishikawa, T. Iino, H. Sawada, T. Shima, E. Ishikawa, A. Imaoka, T. Takahashi, M. Morotomi, and K. Nomoto. 2012. Health-Promoting Effects of B. breve strain Yakult and B. bifidum strain Yakult. Pages 145-298 in Advances in the Science of Bifidobacterium Yakult Strains-Intestinal Microbiota and Human Health. Yakult Central Institute for Microbiological Research, Yakult Honsha Co. Ltd., Tokyo, Japan.

Urita, Y., T. Watanabe, T. Maeda, Y. Sasaki, M. Sugimoto, F. Ishikawa, T. Iino, H. Shibahara-Sone, R. Akahori, S. Mizusawa, A. Nose D. Nozaki, K. Hirano, C. Nonaka, and T. Yokokura. 2008. O040 Bifidobacterium bifidum fermented milk enhances gastric emptying. Clin. Nutr. Suppl. 1:19.

Waller, P. A., P. K. Gopal, G. J. Leyer, A. C. Ouwehand, C. Reifer, M. E. Stewart, and L. E. Miller. 2011. Dose-response effect of Bifidobacterium lactis HN019 on whole gut transit time and functional gastrointestinal symptoms in adults. Scand. J. Gastroenterol. 46:1057-1064

Watanabe, T., Y. Urita, M. Sugimoto, and K. Miki. 2007. Gastroesophageal reflux disease symptoms are more common in general practice in Japan. World J. Gastroenterol. 13:4219-4223.

Yamamoto, Y., S. Sakurabayashi, K. Abe, S. Sezai, M. Hirano, and H. Oka. 1994. Anti-ulcer effect of Bifidobacterium bifidum YIT 4007. Japan. Pharmacol. Therapeut. 22:4743-4746. 